\title{
El uso del smartphone como herramienta para la búsqueda de información en los estudiantes de pregrado de educación de una universidad de Lima Metropolitana
}

CARLOS SAUSSURE FIGUEROA PORTILLA*

Universidad Católica Sedes Sapientiae - Perú

Recibido el 03-11-2015; primera evaluación el 29-11-2015; segunda evaluación el 10-12-2015; tercera evaluación el 26-01-2016; aceptado el 20-04-2016

\section{Resumen}

Los dispositivos móviles como la tablet y el smartphone, sobre todo este último por su portabilidad y fácil acceso a internet, han extendido su uso a un público masivo, dentro del cual se encuentran los estudiantes universitarios.

En el presente artículo se muestran los resultados de una investigación cuantitativa acerca de cómo se realiza la búsqueda de información a través del uso educativo del smartphone por parte de los estudiantes ingresantes del ciclo 2015-I de la Facultad de Educación de una universidad de Lima Metropolitana, de los cuales todos poseen un smartphone.

A fin de obtener la información para el presente estudio, se aplicó una encuesta al grupo señalado. A continuación se presenta la síntesis de los resultados, así como las conclusiones respectivas.

Palabras clave: aprendizaje móvil, teléfono inteligente, búsqueda de información, estudiantes universitarios.

\footnotetext{
Magíster en Integración e Innovación Educativa de la TIC (PUCP), licenciado en Educación Secundaria, en la especialidad de Informática y estudiante de la carrera de Diseńo Gráfico en el Instituto Toulouse Lautrec. Actualmente labora en el Centro de Innovación Educativa - UCSS Virtual de la Universidad Católica Sedes Sapientiae, desempeñando el cargo de diseñador instruccional y locutor profesional. Contacto: csfigueroa@pucp.pe
} 
The use of the smartphone as a tool for the search of information in the undergraduate students of Education of a Metropolitan Lima's university

\section{Abstract}

The mobile devices like the tablet and the smartphone, especially this latter, for its portability and easy access to internet, has allowed its use to a massive public, within which are the university students.

In this article are shown the results of a quantitative research about how is done the information search through the educational use of the smartphone by the incoming students of the 2015-I cycle of the education faculty in a Metropolitan Lima's university, who all of them own a smartphone.

Keywords: mobile learning, smartphone, search of information, university students.

Uso do smartphone como uma ferramenta para encontrar informaçóes sobre estudantes de graduaçáo da faculdade de educaçáo em uma universidade privada em Lima Metropolitano

\section{Resumo}

Dispositivos móveis como a tablet e o smartphone, especialmente o último por sua portabilidade e fácil acesso à internet, têm alargado sua utilizaçáo para uma audiência de massa, como são os estudantes universitários.

Neste artigo sáo apresentados os resultados de uma pesquisa quantitativa sobre como encontrar informaçóes através do uso educacional do smartphone por alunos do Ciclo 2015-I da Faculdade de Educação de uma universidade em Lima, todos os que têm um smartphone.

A fim de obter a informação para este estudo, foi aplicada uma pesquisa ao grupo indicado. Após são apresentadas a síntese dos resultados e também as respectivas conclusôes.

Palavras-chave: aprendizagem móvel, smartphone, pesquisa de informação, estudantes universitários. 


\section{INTRODUCCIÓN Y MARCO TEÓRICO}

Los dispositivos móviles actuales (smartphones, tablets, phablets ${ }^{1}$, etc.) constituyen parte de la tecnología con la que interactuamos en la actualidad. Dispositivos como el smartphone o 'teléfono inteligente' permiten acceder a internet desde cualquier lugar en donde uno se encuentre. Entre las principales definiciones acerca del smartphone, Quicios, Sevillano y Ortega (2013) señalan que se trata de un teléfono móvil que cuenta con un sistema de gestión de la información y características técnicas similares a una laptop; Yu y Conway (2012) indican que un dispositivo móvil como el smartphone tiene las funciones básicas de un teléfono y las mismas capacidades de un computador, con el agregado de la movilidad; por otra parte, Brazuelo y Gallego (2012) señalan que el smartphone es un dispositivo que cuenta con un terminal inteligente con conexión a internet (a través de wifi, $3 \mathrm{G}$ o 4G); finalmente, Organista, Serrano, McAnally y Lavigne (2013) indican que el smartphone es un celular convencional que tiene conectividad avanzada (wifi, 3G/4G) y capacidad para ejecutar aplicaciones.

Considerando los estudios presentados, se puede destacar que el smartphone es un dispositivo móvil que cuenta con las funciones básicas de un teléfono convencional (mensajería de texto, llamadas de voz, etc.); asimismo, permite ingresar a internet y ejecutar aplicaciones, ya que cuenta con un procesador y un sistema operativo que puede ser Android, iOS, Windows, Firefox OS u otro; además, el smartphone tiene capacidades similares a una computadora, con la ventaja de ser portátil y portable.

Por otra parte, según un estudio de Futuro Labs (2014), los jóvenes entre 20 y 29 años (18\% de la población peruana), rango de edad en el que generalmente se encuentran los estudiantes universitarios, hacen uso del smartphone, en mayor porcentaje (85\%), para acceder a redes sociales. En la Universidad de Baja California, México (Organista, Serrano, McAnally \& Lavigne, 2013), muestra que los estudiantes universitarios, usuarios de smartphones, alcanzan un $97 \%$. En otro estudio llevado a cabo en la Universidad de Colorado (Dean, 2010) se encuentra que el 53\% de los encuestados posee un smartphone, que es usado principalmente para mensajería corta y por e-mail $y$, en segundo lugar, como un dispositivo para leer noticias y observar videos en internet.

\footnotetext{
$1 \quad$ Híbrido entre un smartphone y una tablet. Generalmente poseen pantallas entre 5 y 6 pulgadas. Algunos de estos dispositivos cuentan con un lápiz electrónico.
} 
Desde la aparición del smartphone, en el 2008, las capacidades y la usabilidad de este dispositivo se han ido incrementando (Yu \& Conway, 2012), así como su uso masivo a nivel mundial. Un estudio realizado por Canalys Research (Cooper, 2012) señala que, del año 2010 al 2011, el crecimiento de las ventas de smartphones aumentó en $62,7 \%$, frente a un $14,8 \%$ de las PC; es decir, en el 2011 se vendieron más smartphones que computadoras. Según información de la International Data Corporation (IDC, 2014), el mercado de los teléfonos inteligentes o smartphones ha crecido 25,3\% año a año. En el caso de Perú, los estudios realizados por el Organismo Supervisor de Inversión Privada en Telecomunicaciones (Osiptel, 2014) determinaron que en el 2012, el 13,7\% de los peruanos con algún teléfono móvil activo, tenía un smartphone; en el 2013, este porcentaje aumentó a 21,4\%.

El smartphone y otros dispositivos móviles están siendo utilizados recientemente dentro de las aulas de clase, tanto escolares como universitarias. A este uso se le denomina móvil learning, m-learning o aprendizaje móvil. Según Cabero y Castaño (2013, p. 14), las razones por las cuales el aprendizaje móvil está teniendo mayor trascendencia en la educación son las siguientes:

-La portabilidad que están alcanzando las tecnologías.

-La facilidad en su manejo.

-La reducción de los costes de los equipos y de la conexión a Internet.

-El aumento de la conectividad inalámbrica.

-La convergencia funcional que empieza a aparecer entre diferentes dispositivos.

-La rápida adopción de teléfonos inteligentes en nuestra sociedad, y específicamente en el colectivo de profesores y estudiantes.

-La penetración que las últimas generaciones de los dispositivos móviles como las tabletas están alcanzando.

-El aumento del tamaño de la pantalla, pues en la movilidad esta manda.

El aprendizaje móvil es, según Brazuelo y Gallego (2012), una modalidad educativa que, a través del uso de dispositivos móviles, facilita la construcción del conocimiento, la resolución de problemas y el desarrollo de habilidades. Por otra parte, la Unesco (2013) lo define como el uso de tecnología móvil para facilitar el aprendizaje en cualquier momento y lugar.

Asimismo, el smartphone y demás dispositivos móviles permiten a los usuarios consumir información y tener la posibilidad de aprender desde cualquier lugar, lo que impulsa la actual popularidad del aprendizaje móvil. Al respecto, Cabero y Castaño (2013) destacan las siguientes características distintivas de los móviles: la conectividad instantánea (conexión a internet a través de opciones), 
el acceso a diferentes fuentes y tipos de información (acceso multimedia), y la disponibilidad de acceso a la información 24 horas al día durante todo el año. Además, Ozdamli y Cavus (2011) señalan que, para que se lleve a cabo un adecuado aprendizaje a través de los móviles, es necesaria la convergencia de varios elementos, los cuales son el profesor, el estudiante, el contenido, el entorno o el espacio en donde desarrollar el aprendizaje móvil y la evaluación. El estudiante, como centro del proceso de aprendizaje, puede acceder a la información cuando lo requiera, es responsable de su aprendizaje, descubre y hace uso de su estilo, crea y comparte nueva información y estudia con sus compañeros de forma colaborativa. El rol del profesor cambia con el m-learning: ahora el docente es consultor y debe tener la capacidad de identificar los intereses de los estudiantes, así como estar plenamente calificado en el uso de herramientas y tecnologías móviles. El contenido puede ser presentado de diferentes formas y puede variar dependiendo de las necesidades de los alumnos. El entorno de aprendizaje es el lugar donde los estudiantes acceden a la información; para ello es importante recordar que el aprendizaje móvil elimina las limitaciones geográficas, es decir, se puede aprender con estudiantes y/o profesores de otros países sin necesidad de estar presente; esto último ciertamente se asemeja al e-learning, con una característica adicional: la movilidad o ubicuidad (Yu \& Conway, 2012). En relación con el último elemento del aprendizaje móvil, la evaluación, se propone que esta sirva para ayudar a los estudiantes a absolver dudas acerca de la lección impartida y para aprender un poco más de esta, además de servir de diagnóstico formativo del estudiante.

El aprendizaje móvil o m-learning posee un gran potencial como una forma más de poder acercar la educación independientemente de las distancias geográficas. El m-learning es una evolución del e-learning ${ }^{2}$, que, planificándose e integrándose adecuadamente en los entornos educativos, incrementa la calidad del aprendizaje; sin embargo, por ser una tecnología reciente, su uso en entornos académicos aun no es masivo (Yu \& Conway, 2012), al menos no formalmente.

El uso de las tecnologías actuales y dispositivos tecnológicos requiere de un conjunto de habilidades y, como consecuencia, de competencias, las cuales son denominadas competencias digitales, ya que evidencian el conocimiento, habilidades y actitudes que posee el estudiante para utilizar las tecnologías digitales en diversos aspectos a lo largo de toda su vida. Aunque no existen competencias específicas acerca del manejo de dispositivos móviles como el smartphone, las competencias digitales engloban las habilidades para el uso de dispositivos tecnológicos dentro de los cuales, evidentemente, se encuentran los móviles.

2 E-learning es el aprendizaje a distancia mediado por computadoras. 
Diversos países en el mundo proponen estándares de competencias que deben adquirir los estudiantes con respecto al uso de las tecnologías. La Organización para la Cooperación y el Desarrollo Económico (OECD, por sus siglas en inglés), señala en el documento 21st Century skill and competences for new learners in OECD countries (Ananiadou \& Claro, 2009) que las competencias para el siglo XXI pueden ser divididas en tres dimensiones: información, comunicación y ética e impacto social; la primera dimensión incluye dos sub dimensiones: información como recurso (búsqueda, selección, evaluación y organización de la información) e información como producto (reestructuración y el modelado de la información y el desarrollo del conocimiento); la segunda dimensión incluye la comunicación efectiva y la colaboración e interacción virtual, y la tercera dimensión incluye las sub dimensiones de responsabilidad e impacto social.

En el presente estudio se tendrá en cuenta la propuesta hecha por Ferrari (2013) para la Comisión Europea, planteamiento en el que la autora determina cinco áreas: información, comunicación, creación de contenido, seguridad y resolución de problemas. Si bien del área de información se desprende la competencia de navegación, búsqueda y filtro de información, en el presente estudio se analizará específicamente la habilidad de búsqueda de información, que forma parte de la competencia antes indicada.

El área de información está constituida por tres competencias (Ferrari, 2013):

- La primera de estas se refiere a la navegación, búsqueda y filtro de información, que supone acceder y buscar información en internet, encontrar información relevante, seleccionar los recursos de manera eficaz, navegar entre los recursos en línea y crear estrategias de información personal. La competencia navegación, búsqueda y filtro de información tiene tres niveles de dominio: el inicial, cuando el estudiante puede realizar investigaciones online a través de los diversos motores de búsqueda y además es consciente de que diferentes motores de búsqueda arrojan resultados distintos; el nivel intermedio, cuando, además de realizar las acciones señaladas en el nivel inicial, el estudiante puede seleccionar adecuadamente la información que encuentra; el nivel avanzado, cuando el estudiante puede utilizar un amplio rango de estrategias de búsqueda al investigar y navegar por la web.

- La segunda competencia se refiere a la evaluación de la información, la cual supone reunir, procesar y evaluar críticamente la información. Esta competencia tiene, a su vez, tres niveles de dominio: el inicial, cuando el estudiante es consciente de que no toda la información que existe en 
la web es confiable; el nivel intermedio, cuando compara diversos recursos de información y el nivel avanzado, cuando el estudiante es crítico respecto de la información que encuentra y es capaz de cotejar su validez y su credibilidad.

- Finalmente, la tercera competencia es el almacenamiento y la recuperación de información, la cual se refiere a saber manipular y almacenar información y contenidos para su fácil recuperación, y para organizar la información y los datos obtenidos. Esta competencia tiene tres niveles de dominio: inicial, cuando el estudiante conoce cómo guardar archivos y contenidos, como textos, gráficos, música, video, etc., y sabe también cómo regresar al espacio donde los guardó; el nivel intermedio, cuando el estudiante puede guardar, almacenar o etiquetar archivos y desarrollar su propia estrategia de almacenamiento y, además, puede administrar la información y el contenido guardado o almacenado; el nivel avanzado, cuando el estudiante aplica diferentes métodos y herramientas para organizar los archivos o la información, y además puede emplear diversas estrategias para recuperar los contenidos que él u otros estudiantes han organizado.

Año tras año, las fuentes de información, como bibliotecas digitales, bases de datos, portales web, blog, entre otras, se amplían y son cada vez más numerosas, por lo que tiene prioridad que el estudiante universitario, que hace uso de tecnologías móviles como el smartphone, disponga de capacidades y habilidades que, como señala Area (2010), le permitan la búsqueda de información especializada en bases de datos relevantes para su labor académica.

Por lo tanto, de acuerdo con lo expuesto, se planteó la siguiente pregunta de investigación:

¿Cómo se realiza la búsqueda de información a través del uso educativo del smartphone por parte de los estudiantes de pregrado de la Facultad de Educación de una universidad de Lima Metropolitana?

El objetivo general de la investigación fue el siguiente:

Determinar cómo los estudiantes de pregrado de la Facultad de Educación, de una universidad de Lima Metropolitana, usan educativamente el smartphone a fin de buscar información para sus actividades académicas.

A partir de este objetivo general, se plantearon los siguientes objetivos específicos:

1. Identificar los usos educativos del smartphone como herramienta para la búsqueda de información.

2. Determinar qué tipo de aplicaciones y páginas web utilizan, con mayor frecuencia, para la búsqueda de información a través del smartphone. 
3. Identificar la opinión que tienen sobre la adquisición de habilidades de búsqueda de información desde que hacen uso del smartphone para sus actividades educativas.

\section{Metodología}

El presente es estudio de tipo cuantitativo exploratorio (Hernández, Fernández \& Baptista, 2013), ya que tiene como finalidad recoger información acerca de cómo los estudiantes de pregrado de la Facultad de Educación de una universidad de Lima Metropolitana obtienen información al hacer uso del smartphone en sus actividades educativas, información que fue sistematizada haciendo uso de porcentajes.

\section{Población y muestra}

La población estuvo conformada por la totalidad de estudiantes ingresantes (74) a la Facultad de Educación, que cursaban el ciclo 2015-I en una universidad privada de Lima Metropolitana. La población de estudiantes ingresantes a la Facultad de Educación fue de 74 estudiantes, 60 de los cuales formaron parte del estudio por cumplir con el criterio de poseer un smartphone.

\section{Mediciones e instrumentos}

A fin de identificar cómo los estudiantes de pregrado de la Facultad de Educación de una universidad de Lima Metropolitana obtienen información al hacer uso del smartphone en sus actividades académicas, se elaboró una encuesta como instrumento cuantitativo de recolección de datos (Hernández, Fernández \& Baptista, 2013). Dicho instrumento fue sometido a la validación de expertos (Hernández, Fernández \& Baptista, 2013) y su aplicación se realizó de forma presencial.

La encuesta constó de nueve preguntas cuya variable fue la búsqueda de información, constituida por las siguientes subvariables: uso de aplicaciones para la búsqueda de información, formato de búsqueda de información y percepción de los estudiantes sobre la adquisición de habilidades para la búsqueda de información al hacer uso del smartphone en sus actividades educativas. 


\section{Resultados}

A partir de la encuesta realizada y de acuerdo con el orden de las preguntas se destacan los siguientes resultados.

Tabla 1. Frecuencia de uso de navegadores, redes sociales, buscadores de videos y portales de noticias

\begin{tabular}{|l|c|c|c|}
\hline & Siempre & A veces & Nunca \\
\hline $\begin{array}{l}\text { Aplicaciones de navegador a fin de buscar información } \\
\text { para las actividades educativas }\end{array}$ & \multicolumn{3}{|l|}{} \\
\hline Google Chrome & $81,4 \%$ & $15,3 \%$ & $3,4 \%$ \\
\hline Safari & $6,7 \%$ & $11,7 \%$ & $81,7 \%$ \\
\hline Mozilla Firefox & $8,3 \%$ & $25 \%$ & $66,7 \%$ \\
\hline Otros & $3,4 \%$ & $13,6 \%$ & $83,1 \%$ \\
\hline $\begin{array}{l}\text { Aplicaciones de redes sociales a fin de buscar informa- } \\
\text { ción para las actividades educativas }\end{array}$ & \multicolumn{3}{|l|}{} \\
\hline Facebook & $50 \%$ & $38,3 \%$ & $11,7 \%$ \\
\hline Twitter & $1,7 \%$ & $28,3 \%$ & $70 \%$ \\
\hline Instagram & $15 \%$ & $20 \%$ & $65 \%$ \\
\hline Pinterest & $5 \%$ & $15 \%$ & $80 \%$ \\
\hline Otros & $13,6 \%$ & $23,7 \%$ & $62,7 \%$ \\
\hline Página web y aplicación de Youtube & $31,7 \%$ & $43,3 \%$ & $25 \%$ \\
\hline Página web de Youtube & $55 \%$ & $35 \%$ & $10 \%$ \\
\hline Aplicación de Youtube & \multicolumn{3}{|l}{} \\
\hline Página web y aplicación de portales de noticias & $18,3 \%$ & $45 \%$ & $36,7 \%$ \\
\hline Aplicaciones de portales de noticias & $36,7 \%$ & $41,7 \%$ & $21,7 \%$ \\
\hline Páginas web de portales de noticias &
\end{tabular}

En relación con la frecuencia de uso de diversos tipos de aplicaciones de navegadores a fin de buscar información para las actividades educativas, los estudiantes señalaron a Google Chrome como la aplicación de navegador más usada $(81,4 \%)$.

Respecto del uso de aplicaciones de redes sociales para la búsqueda de información para actividades educativas, el 50\% de los estudiantes respondió que siempre hace uso de la aplicación Facebook para tales propósitos. 
En relación al servicio de videos de Youtube, tanto en su versión de aplicativo como en la versión web, el 31,7\% de los estudiantes respondió que siempre utiliza la página web de Youtube para buscar información (videos) y, por otra parte, el 55\% respondió que también utiliza siempre la aplicación de Youtube. Este resultado hace evidente que la mayor frecuencia de uso de la aplicación de Youtube para la búsqueda de información se corresponde con la naturaleza del smartphone como dispositivo que ejecuta aplicaciones.

Finalmente, respecto del uso de aplicaciones o páginas web de portales de noticias para la búsqueda de información a través del smartphone, el 18,3\% de estudiantes señaló que siempre utiliza aplicaciones de portales de noticias, mientras que el 36,7\% indicó que nunca usa ese tipo de aplicaciones. En cuanto a la frecuencia de uso de páginas web de portales de noticias para la búsqueda de información para actividades educativas, el 36,7\% de los estudiantes encuestados respondió que siempre utiliza ese tipo de páginas web.

Tabla 2. Habilidades y nivel de dominio de la competencia de búsqueda de información

\begin{tabular}{|l|c|c|}
\cline { 2 - 3 } \multicolumn{1}{l|}{} & Sí & No \\
\hline Sé que existen diversos motores de búsqueda & $83,3 \%$ & $16,7 \%$ \\
\hline Hago algunas búsquedas en línea a través de motores de búsqueda & $66,7 \%$ & $33,3 \%$ \\
\hline $\begin{array}{l}\text { Sé que los diferentes motores de búsqueda arrojan diferentes } \\
\text { resultados }\end{array}$ & $76,7 \% \%$ & $23,3 \% \%$ \\
\hline Puedo organizar la información que necesito buscar & $81,7 \%$ & $18,3 \%$ \\
\hline $\begin{array}{l}\text { Al buscar información en internet utilizo un amplio rango de } \\
\text { estrategias de búsqueda }\end{array}$ & $65 \%$ & $35 \%$ \\
\hline $\begin{array}{l}\text { Sé cuál (cuáles) es (son) el (los) motor(es) de búsqueda que arro- } \\
\text { jará (n) la mejor información que necesito }\end{array}$ & $63,3 \%$ & $36,7 \%$ \\
\hline Busco la información presentada mediante links o enlaces & $75 \%$ & $25 \%$ \\
\hline
\end{tabular}

En relación con las habilidades y nivel de dominio de la competencia de búsqueda de información, los estudiantes respondieron que conocen la existencia de diversos motores de búsqueda $(83,3 \%)$, que realizan búsqueda de información a través de diversos motores de búsqueda $(66,7 \%)$, que saben que cada motor de búsqueda arroja un resultado distinto $(76,7)$ y, además, que realizan búsqueda por páginas web a través de enlaces (75\%). 
El 53,3\% de los estudiantes encuestados señaló que cada día o casi a diario busca información escrita (noticias, artículos, tesis, entre otros), mientras que el 35\% realiza este tipo de actividad dos o tres veces a la semana.

En relación con la búsqueda de información con imágenes (gráficos o fotos), el $48 \%$ los estudiantes encuestados señaló que realiza esta actividad cada día o casi a diario, mientras que el 36,7\% indicó que lo hace dos o tres veces a la semana.

Tabla 3. Frecuencia de búsqueda de información en formatos de texto, imagen, video y audio

\begin{tabular}{|l|c|c|c|c|c|}
\cline { 2 - 6 } \multicolumn{1}{c|}{} & $\begin{array}{c}\text { Cada día o } \\
\text { casi a diario }\end{array}$ & $\begin{array}{c}\text { Dos o tres veces } \\
\text { a la semana }\end{array}$ & $\begin{array}{c}\text { Dos o tres } \\
\text { veces al mes }\end{array}$ & $\begin{array}{c}\text { Menos de una } \\
\text { vez al mes }\end{array}$ & Nunca \\
\hline $\begin{array}{l}\text { Formato } \\
\text { textual }\end{array}$ & $53,3 \%$ & $35 \%$ & $6,7 \%$ & $1,7 \%$ & $3,3 \%$ \\
\hline $\begin{array}{l}\text { Formato } \\
\text { de imagen }\end{array}$ & $48,3 \%$ & $36,7 \%$ & $8,3 \%$ & $5 \%$ & $1,7 \%$ \\
\hline $\begin{array}{l}\text { Formato } \\
\text { de video }\end{array}$ & $43,3 \%$ & $30 \%$ & $15 \%$ & $6,7 \%$ & $5 \%$ \\
\hline $\begin{array}{l}\text { Formato } \\
\text { de audio }\end{array}$ & $35 \%$ & $35 \%$ & $15 \%$ & $11,7 \%$ & $3,3 \%$ \\
\hline
\end{tabular}

Acerca de la búsqueda de información en formato de video, el 43,3\% de los estudiantes encuestados señaló que realiza esta actividad casi a diario, mientras el $30 \%$ la lleva a cabo dos o tres veces a la semana.

$\mathrm{Al}$ ser consultados acerca de la frecuencia de búsqueda de información en formato de audio (música o grabaciones de voz) para actividades con fines educativos, el 35\% de los estudiantes encuestados señaló que realiza la búsqueda cada día o casi a diario, y un porcentaje similar de estudiantes la lleva a cabo dos o tres veces por semana.

Así pues, en cuanto a la información presentada en los cuatro cuadros anteriores acerca de la frecuencia diaria o casi diaria respecto de la búsqueda de información, se observa que el mayor porcentaje corresponde al formato textual (noticias, artículos, tesis, entre otros): 53,3\%; en segundo lugar se encuentra la búsqueda de información en formato de imágenes: 48,3\%; en tercer lugar se ubica la búsqueda de información en formato de video: $43,3 \%$; en cuarto lugar se halla la búsqueda de información en formato de audio: 35\%. 
Por último, se consultó si los estudiantes eran conscientes de la adquisición de habilidades para buscar información desde que usan el smartphone a fin de desarrollar sus actividades educativas. Los resultados fueron los siguientes:

\section{Tabla 4. ¿Consideras que has adquirido habilidades para buscar información desde que usas tu smartphone con la finalidad de desarrollar tus actividades educativas?}

\begin{tabular}{|l|c|}
\hline De acuerdo & $56,7 \%$ \\
\hline Medianamente de acuerdo & $38,3 \%$ \\
\hline En desacuerdo & $5,0 \%$ \\
\hline
\end{tabular}

Como se puede observar, los estudiantes encuestados consideraron, en su mayoría (56,7\%), que adquirieron habilidades para buscar información desde que usan el smartphone en sus actividades educativas.

\section{Discusión de resultados}

Los resultados del presente estudio indican que las características principales que los estudiantes consideran necesarias en un smartphone a fin de que sea utilizado como herramienta para la búsqueda de información en sus actividades educativas son: el fácil acceso a internet (plan de datos o conexión wifi) y la duración de la batería, lo cual no deja de ser coherente, debido a que al hacer uso del smartphone, a fin de buscar información, lo más importante es que este tenga acceso a redes de internet, así como también es importante que la duración de la batería sea bastante amplia, puesto que el uso constante de internet consume la carga energética del dispositivo móvil.

Estos resultados coinciden con los obtenidos en la investigación hecha por Taleb y Sohrabi (2012) en la que se evidencia que, entre los estudiantes de la Islamic Azad University of South Tehran Branch, los factores más importantes para utilizar educativamente el smartphone son que presente diversas capacidades, que cuente con una larga duración de la batería y que tenga fácil acceso a internet. Ante lo hallado en la presente investigación se puede afirmar que el uso educativo, y más específicamente la búsqueda de información a través del smartphone, depende mucho de factores como la duración de la batería y la facilidad de acceso a internet. Por otra parte, los datos obtenidos por Taleb y Sohrabi (2012) señalan que el principal uso que los alumnos hacen del teléfono móvil son como calculadora $(21,3 \%)$ y el uso menos frecuente es como herramienta para buscar material educativo con internet $(1,14 \%)$; frente a los resultados obtenidos en el presente estudio, en el que los datos arrojaron que 
los estudiantes utilizan siempre aplicaciones como Google Chrome (81,4\%), Facebook (50\%) y Youtube (55\%) para realizar búsqueda de información a través de su smartphone, hallazgos que se apoyan en lo investigado por Henríquez, Organista y Lavinge (2013), así como Organista, McAnally y Lavinge (2013). En el primer estudio, el 60,6\% de los encuestados utiliza el smartphone para la búsqueda, intercambio y descarga de información de internet; en el segundo estudio, el $42 \%$ de estudiantes encuestados utiliza aplicaciones de buscadores de información en su smartphone. Así pues, se debe señalar que las diferencias encontradas entre la investigación de Taleb y Sohrabi (2012) y la presente investigación se deberían a que aquella se enfocó en el uso de móviles en general: tanto smartphones y no smartphones. Como lo evidencia la investigación hecha por Quicios, Sevillano y Ortega (2013), los estudiantes que poseen móviles (no smartphones) hacen uso de estos principalmente para mensajería instantánea (SMS) y captura de imágenes por la cámara de fotos; en cambio, quienes poseen smartphones realizan comúnmente actividades como ingresar al campus virtual, acceder a páginas web, usar el smartphone como una computadora, entre otros.

Asimismo, los resultados de la presente investigación destacan que, en su mayor parte, los estudiantes encuestados consideraron que han adquirido habilidades para la búsqueda de información desde que hacen uso del smartphone como herramienta educativa, lo cual coincide con lo investigado por Quicios, Sevillano y Ortega (2013), en cuyo estudio se evidencia que el $28 \%$ de estudiantes considera que ha adquirido habilidades de manejo de información a través del uso académico de los smartphones.

Por otra parte, de acuerdo con los indicadores propuestos por Ferrari (2013) para identificar el nivel de adquisición de la competencia de navegación, filtro y búsqueda de información, y en concordancia con los hallazgos de la presente investigación, se puede afirmar que, según el porcentaje correspondiente a las respuestas dadas frente al conocimiento de la existencia de diversos motores de búsqueda $(83,3 \%)$, se puede afirmar que los estudiantes han alcanzado el nivel inicial de dominio de la competencia navegación, filtro y búsqueda de información; asimismo, el porcentaje de respuestas positivas obtenidas respecto de hacer búsquedas en línea a través de motores de búsqueda $(66,7 \%)$ y poder organizar la información que necesitan buscar $(81,7 \%)$ da cuenta de que los estudiantes encuestados han alcanzado, también, el nivel intermedio de dominio de la competencia de navegación, filtro y búsqueda de información; además, el mayor porcentaje positivo de respuestas obtenidas acerca del manejo de un amplio rango de estrategias de búsqueda (65\%) denota que los estudiantes están alcanzando, también, un nivel de dominio avanzando de la competencia de navegación, filtro y búsqueda de información al hacer uso del smartphone. 


\section{Conclusiones}

Los resultados del presente estudio evidencian la importancia del smartphone como herramienta para la búsqueda de información académica en los estudiantes universitarios encuestados. Además, los resultados obtenidos muestran que el tipo de información que buscan los estudiantes es, en mayor porcentaje, la información textual $(53,3 \%)$, seguida de la información en formato de imagen (48,3\%); mientras que la información en formato de audio es la que menos buscan $(35 \%)$, por lo que se puede afirmar que recursos como los podcast son poco conocidos por los estudiantes.

Respecto de la frecuencia de uso de aplicaciones, se manifiesta una amplia tendencia al uso de Google Chrome (81,4\%) y, en consecuencia, del motor de búsqueda de Google, para realizar búsquedas con fines académicos, lo cual se corresponde con los hallazgos señalados respecto del tipo de formato de información que los estudiantes buscan con mayor frecuencia, ya que Google muestra, principalmente, resultados de información escrita. Respecto de la red social de preferencia para la búsqueda de información, los estudiantes señalaron a Facebook como la principal (50\%); este resultado no solo hace evidente una creciente tendencia de los estudiantes a utilizar el recurso que brinda la red social como grupos, mensajería instantánea (chats) o páginas web, sino que también puede ser de utilidad para destacar los usos que pueden tener las redes sociales dentro de las actividades académicas de los estudiantes.

Estos hallazgos, así como lo mostrado en las tabla 1 acerca de la mayor frecuencia de uso de páginas web como Google y Youtube para la búsqueda de información, son además relevantes porque Google y Youtube cuentan con un sistema predictivo de búsqueda, el cual sugiere palabras clave al usuario mientras este digita un texto en la barra de búsqueda, lo cual podría favorecer también la adquisición de habilidades.

Es importante explicitar que los resultados fueron positivos en cuanto a demostrar la percepción de los estudiantes sobre el desarrollo de habilidades de búsqueda de información al hacer uso del smartphone; por lo que, de estar contemplado su uso como parte de la planificación curricular de la universidad, las competencias adquiridas por parte de los estudiantes serían mucho mayores.

Cabe señalar que la investigación ha sido realizada a una muestra de estudiantes de educación y que la aplicación de la encuesta a un grupo diferente, tanto en cantidad como en diversidad de facultades, podría modificar los resultados mostrados. 
Finalmente, la investigación presentada es un aporte para estudios posteriores en los que se busque sustentar la utilidad educativa no solo de los smartphones sino también de otros dispositivos móviles como las tablet.

\section{REFERENCIAS BIBLIOGRÁFICAS}

Ananiadou, K. \& Claro, M. (2009). 21st century skills and competences for new millennium learners in OECD countries, OECD education working papers no. 41. París: OECD.

Area, M. (2010). ¿Por qué formar en competencias informaciones y digitales en la educación superior? Revista de Universidad y Sociedad del Conocimiento (RUSC), 7(2), 2-4. Recuperado el 20 de 07 de 15, de http://rusc.uoc.edu/ ojs/ index.php/rusc/article/view/v7n2-area/v27n2-area

Brazuelo, F. \& Gallego, D. (2012). Mobile Learning: Dispositivos móbiles como recurso educativo. Bogotá: MAD S.L.

Cabero, J. \& Castaño, C. (2013). Enseñar y aprender en entornos m-learning. Madrid: Síntesis.

Cooper, D. (2012) Engadget. Obtenido de http://www.engadget.com/2012/02/03/ canalys-more-smartphones-than-pcs-shipped-in-2011/

Dean, J. (2010). Digital Media Test Kitchen. Obtenido de http://testkitchen.colorado.edu/reports/smartphone/smartphone-survey/

Ferrari, A. (2013). DIGI COMP: A framework for developing and understanding digital competence in Europe. Sevilla: Joint Research Centre.

Futuro Labs (15 de julio de 2014). Jóvenes Millennials - Perfiles, hábitos y actitudes en el uso del smartphone. Obtenido de http://www.futurolabs.com/ los-millennials-de-lima-y-el-uso-del-smartphone/\#prettyPhoto

Henríquez Ritchie, P., Organista Sandoval, J. \& Lavigne, G. (2013). Nuevos procesos de interactividad e interacción social: uso de smartphones por estudiantes y docentes universitarios. Revista electrónica Actualidades Investigativas en Educación, 13(3), 1-21.

Hernández, R., Fernández, C. \& Baptista, P. (2013). Metodología de la Investigación. México D. F.: Mc Graw Hill.

International Data Corporation. (2014). IDC. Obtenido de http://www.idc.com/ prodserv/smartphone-os-market-share.jsp

Organismo Supervisor de la Inversión Privada en Telecomunicaciones (Osiptel). (2014). Caracterización de la demanda de telefonía móvil en el Perú: 20122013, un estudio descriptivo. Huancayo: Editora Imprenta Ríos SAC. 
Organista Sandoval, J., McAnally Salas, L. \& Lavigne, G. (2013). El teléfono inteligente (smartphone) como herramienta pedagógica. Apertura, 6-19.

Organista, J., Serrano, A., McAnally, L. \& Lavigne, G. (2013). Apropiación y usos educativos del celular por estudiantes y docentes universitarios. REDIE, 139-156.

Organización de las Naciones Unidas para la Educación, la Ciencia y la Cultura (Unesco). (2013). UNESCO Policy Guidelines for Mobile Learning. París: Unesco.

Ozdamli, F. \& Cavus, N. (2011). Basic elements and characteristics of mobiles learning. Procedia: Social and Behavioral Sciences, 28, 937-942.

Quicios, M., Sevillano, M. \& Ortega, I. (2013). Educational Uses of Mobile Phone by University Students in Spain. The New Educational Review, 34(4), 151-163.

Taleb, Z. \& Sohrabi, A. (2012). Learning on the move: the use of mobiles technology to support learning for university students. Procedia: Social and Behavioral Sciences, 69, 1102-1109.

Yu, F. \& Conway, A. R. (2012). Mobile/Smartphone Use in Higher Education. Proceedings of the 2012 Southwest Decision Sciences Institute, 831-839. 